\title{
Validación de una Encuesta de Actitudes de Lectura en estudiantes de Educación Básica ${ }^{*}$
}

\author{
Liliana Fuentes ${ }^{* *}$ \\ María Constanza Errázuriz ${ }^{* * *}$ \\ Omar Antonio Davison ${ }^{* * * *}$ \\ Andrea Cocio ${ }^{* * * * *}$
}

\begin{abstract}
Resumen
Las actitudes de lectura de los estudiantes de educación básica han demostrado ser relevantes en su desempeño lector, por lo que medirlas es fundamental para elevarlo, no obstante, existen pocos instrumentos validados en Chile que permitan relevar este fenómeno. El objetivo del presente estudio fue adaptar y validar el instrumento sobre actitudes hacia la lectura en niños de $4^{\circ}$ a $6^{\circ}$ Básico. Con respecto al método, el diseño fue de tipo evaluativo y descriptivo, el procedimiento cuantitativo y la muestra fue representativa y correspondió a 1.190 estudiantes de $4^{\circ}$ a $6^{\circ}$ Básico con los resultados se confirmó que las dimensiones del instrumento y de los ítems de cada una.
\end{abstract}

Palabras clave: actitudes de lectura, lectura, comprensión lectora, educación escolar.

\section{Validation of a Reading Attitudes Survey in Primary students}

\begin{abstract}
The reading attitudes of primary students have proven to be relevant in their reading performance, so measuring them is fundamental to raise the level. The objective of this study was to validate and adapt the instrument towards reading attitudes in children from 4th, 5 th and 6th grade. Regarding the method, the design was quantitative and descriptive. Regarding the results, it was possible to confirm satisfactorily the dimensions of the instrument and the items for each one of them.
\end{abstract}

Key words: reading attitudes, reading, reading comprehension, school education.

Recibido: 11/10/2017

Aceptado: 29/10/2018

Este artículo se inscribe en los proyectos de investigación Concepciones sobre lectura de profesores en ejercicio: Perfiles lectores de docentes y su relación con el desempeño de sus estudiantes (Código Proyecto: Fonide FX11619) y Concepciones sobre la lectura y su fomento de profesores de Educación Básica en ejercicio y sus estudiantes ¿Cómo inciden en las prácticas docentes?: Análisis de una relación significativa para el desarrollo de lectura en la escuela (Conicyt Fondecyt Regular 1170193).

Chilena. Doctora en Lingüística de la Pontificia Universidad Católica de Valparaíso. Académica Universidad de Chile, Santiago, Chile. lilyfuentesm@gmail.com

*** Chilena. Doctora en Filología Hispánica de la Universdad de Valladolid. Académica Pontiticia Universidad Católica de Chile, Santiago, Chile. cerrazuc@uc.cl

Chileno. Doctor (c) en Ciencias Humanas de la Universidad Austral de Chile. Becario Conicyt (21130086) Programa de Doctorado en Ciencias Humanas Universidad Austral de Chile, Valdivia, Chile. omardavisont@gmail.com

* Chilena. Doctora en Innovación Educativa y Aprendizaje de la Universidad de Deusto. Académica Universidad Católica de Temuco, Temuco, Chile. acocio@uct.cl 


\section{Introducción}

La preocupación por el hábito lector y su fomento está cobrando cada vez más relevancia desde diversas matrices disciplinarias. La promulgación, en Chile, del Plan Nacional de la Lectura (2015-2020) (Consejo Nacional de la Cultura y las Artes, 2015) ratifica el interés de la política pública por posicionar a la lectura como un derecho social en el marco de promover el desarrollo de una sociedad lectora. En ese contexto, cabe indagar en el rol que debe cumplir la escuela y sus docentes frente a los desafíos que supone la formación de alumnos más vinculados con la lectura.

En este sentido, estudios realizados en el país evidencian, por ejemplo, que los adolescentes no manifiestan como actividad primordial la lectura y solo un $19 \%$ se autopercibe como lector frecuente, aun cuando la lectura de 15 a 20 minutos en forma diaria tiende a aumentar conforme avanza la edad (Consejo Nacional de la Cultura y las Artes, 2014). Por su parte, Latorre (2014) concluye que a medida que el estudiantado avanza de grado, la lectura es percibida como menos entretenida y con un fuerte carácter de obligatoriedad.

De hecho, con respecto al desempeño, según la medición internacional PISA 2012, Chile obtuvo 441 puntos en lectura; si bien existe un alza, estamos bajo el promedio de los países de la OCDE que tienen 496 puntos (OCDE, 2014). Esto se agudiza en la región de la Araucanía, Chile, donde se focaliza esta investigación, ya que si bien su puntaje en la evaluación Simce (Sistema de Medición de la Calidad de la Educación) de Lectura de 4º Básico 2017 (266 ptos.) ha subido respecto de los años anteriores, continúa siendo más bajo que el promedio nacional (Chile. Agencia de Calidad de la Educación, 2017). En efecto, una aplicación del test de comprensión lectora Clip a 2.277 estudiantes de esta región, evidenció niveles de comprensión que no superan el 50\% en un nivel superficial o el $20 \%$ en niveles de comprensión profunda (Fuentes, 2009). Probablemente, estos resultados se vinculan con el hecho de que esta zona presenta los mayores índices de pobreza del país (Chile. Ministerio de Desarrollo Social, 2014) y una realidad intercultural que no ha sido suficientemente abordada (Becerra-Lubies \& Mayo, 2015).

Asimismo, la Prueba PISA también confirma la brecha de género en lectura entre alumnos y alumnas, pues estas últimas los superan de manera significativa, lo que también es ratificado por los últimos 
resultados del Simce de Escritura 2016 y por la evidencia internacional (Logan \& Johnston, 2010; Schaffner, Schiefele \& Ulferts, 2013; Stutz, Schaffner \& Schiefele, 2016). Del mismo modo, el Estudio Internacional de Lectura PIRLS 2016 (Chile. Agencia de Calidad de la Educación, 2017a y 2017b) señala que el nivel de actitudes de lectura positivas del estudiantado chileno es menor al promedio internacional.

Por otra parte, existen evidencias que relevan la responsabilidad del profesor como lector frecuente y generador de prácticas lectoras más motivantes con sus estudiantes (Fundación La Fuente, 2016). En ese sentido, el fomento lector en la escuela, asumido como una intervención sistemática que comprende las prácticas pedagógicas que favorecen la lectura, los momentos para su realización, la creación de un ambiente adecuado y las formas de evaluarla e integrarla a la gestión pedagógica, se presenta como una herramienta clave para promover una cultura lectora al interior de las comunidades educativas (Chambers, 2007; Errázuriz, Becerra, Cocio, Davison \& Fuentes, 2018; Lockwood, 2011).

En consecuencia, el propósito de este trabajo es abordar este problema desde el foco del estudiante, específicamente, aportar respecto de uno de los aspectos relevantes en los resultados de comprensión lectora y de producción de textos, esto es, las actitudes de los alumnos hacia estas actividades, las que develan su motivación y pueden predecir su conducta y sus prácticas lectoras (Mújica, Guido \& Mercado, 2011). Punto particularmente sensible, considerando que, según algunas evidencias (Errázuriz et al., 2018; OECD, 2014; Orellana, 2018; Stutz et al., 2016), los estudiantes que muestran actitudes positivas hacia la lectura tienden a ser buenos lectores y alcanzan un mayor rendimiento académico. Especialmente considerando que el desarrollo de la lectura en los contextos escolares puede contribuir a la inclusión de los estudiantes más desaventajados, de modo de superar sus limitaciones relacionadas con el capital cultural y el origen social (Cervini, 2002). Frente a este escenario, es prioritario contar con instrumentos válidos para poder identificar las actitudes de lectura de los estudiantes de escuelas de la Araucanía y, de esta manera, comprender el problema y poder diseñar e implementar políticas públicas y medidas acordes para fomentar y mediar la lectura adecuadamente.

En este sentido, si bien existen numerosos instrumentos sobre actitudes de lectura validados en el mundo anglosajón, hay pocos en español y menos aún validados en el contexto chileno. Por ejemplo, 
la Escala de Motivación de la Lectura Académica de Eccles y Wigfield (2002), validada en Chile por Muñoz et al. (2012), solo aborda la lectura académica no recreativa y fue aplicada y validada en estudiantes universitarios. A su vez, si bien la Escala Motivación por la Lectura (Gambrell, Palmer, Codling \& Mazzoni, 1996) fue validada recientemente en Chile por Navarro, Orellana y Baldwin (2018) en alumnos del ámbito escolar, cabe advertir que su muestra correspodió a sujetos de la Región Metropolitana y de establecimientos particulares pagados y subvencionados, un contexto muy alejado de la realidad de las escuelas municipales de la región de la Araucanía. Además, dicha validación fue la primera aplicación de la escala en hablantes nativos del español, a diferencia de Encuesta de Actitudes hacia la Lectura (Merisuo-Storm \& Soininen, 2012), instrumento seleccionado, que ya cuenta con una validación anterior en estudiantes españoles (García-Rodicio, IzquierdoMagaldi, Fernández-Gutiérrez \& Villalón, 2015).

\section{Marco teórico}

\subsection{Actitudes hacia la lectura}

La actitud hacia la lectura ha sido definida como la predisposición de responder de manera favorable o desfavorable respecto de una actividad de lectura y, también, como un sistema de sentimientos respecto de la lectura que causan que el sujeto se aproxime o evite una situación de lectura, dentro de un continuum con extremos positivos y negativos (Alexander \& Filler, 1976; McKenna, Kear \& Ellsworth, 1995). Es decir, es la imagen que el lector tiene sobre sus interacciones con la lectura de textos. Para Mathewson (1994), la actitud es un factor preponderante durante la lectura y su aprendizaje y se compone de cuatro factores centrales: los valores, los objetivos, el autoconcepto y la retroalimentación cognitiva y afectiva en una experiencia de lectura. Mientras que el modelo de McKenna, Kear y Ellsworth (1995) integra el aspecto social y considera la actitud como un fenómeno de naturaleza afectiva, cuyas creencias se relacionan causalmente con esta y se compone de tres factores principales: las creencias sobre los resultados de lectura según su deseabilidad, las creencias sobre las expectativas de los demás y la motivación por satisfacerlas y los resultados acerca de incidentes de lectura específicos. 
De acuerdo con estudios internacionales como PISA, los estudiantes que muestran actitudes positivas hacia la lectura tienden a ser buenos lectores (OECD, 2014; Villalón, Merisuo-Storm, Izquierdo-Magaldi, Melero \& Soininen, 2016) y tener un mayor rendimiento académico, pues la lectura es una herramienta de aprendizaje en todas las disciplinas (Guthrie et al., 2007). De hecho, Mujica, Guido y Mercado (2011) constataron que las actitudes lectoras de estudiantes mexicanos, especialmente en sus componentes afectivo y cognitivo, influían en su comportamiento lector, lo que se sustenta en las evidencias que existen sobre cómo las actitudes se relacionan estrechamente con la conducta de las personas (Azjen \& Fishbein, 1977; Carpi \& Breva, 2001). Incluso, también hay algunas investigaciones que señalan factores de género a favor de las mujeres (Calderón, 2015; Coles \& Hall, 2002; Logan \& Johnston, 2010; Mullis, Martin, Kennedy \& Foy, 2007; Schaffner et al., 2013; Stutz et al., 2016), quienes obtienen mejores puntajes que los varones en comprensión lectora y mantienen actitudes positivas hacia esta, como demuestran los resultados del Simce.

En relación con las evidencias sobre las actitudes de lectura de alumnos de educación primaria, un estudio realizado a 18.185 niños estadounidenses de $1^{\circ}$ a $6^{\circ}$ grado (McKenna, Kear \& Ellsworth, 1995), constató que las actitudes hacia la lectura académica y recreativa al comienzo de la escolaridad eran relativamente positivas, pero que terminaban en total indiferencia en sexto grado y que no había diferencias según la etnia, pero sí de género a favor de las niñas. De este modo, los lectores más débiles presentaron actitudes más negativas que los mejores lectores, por lo que se comprobó que la actitud afectaba la habilidad de lectura y esta relación se acrecentaba con el tiempo.

En efecto, pareciera ser que las actitudes hacia la lectura se desarrollan o decaen mayormente en la escuela (Villalón et al., 2016), pues son influenciadas por los perfiles lectores y prácticas docentes de sus profesores. De este modo, Daisey (2009), Granado (2014) y Morrison, Jacobs y Swinyard, (1999) descubrieron que los docentes que disfrutan de la lectura en su vida pueden proyectar y recrear estas experiencias positivas con sus estudiantes en el aula escolar (Jiménez et al., 2015).

Asimismo, según otros estudios (Martínez, Aricak \& Jewell, 2008; Petscher, 2010), las actitudes de alumnos estadounidenses demostraron volverse negativas de modo gradual a lo largo de los niveles de la escuela 
y esta negatividad se incrementó en estudiantes con dificultades; por el contrario, las niñas se mostraron más positivas hacia esta labor. Además, nuevamente se confirmó que la actitud predecía el desempeño lector. Del mismo modo, Guthrie et al. (2007), Van Schooten, De Glopper y Stoel (2004) y Wigfield et al. (2008) también comprobaron que una mayor motivación por la lectura de estudiantes de educación primaria y secundaria predecía un mayor crecimiento de la habilidad de comprensión lectora.

Por su parte, estudiantes de primer y segundo año de primaria de España y Finlandia mostraron tener actitudes más positivas hacia la lectura en primer año que en segundo grado y también las niñas presentaron mayor motivación por leer (Villalón et al., 2016), sin embargo, no se identificó que hubiese relación entre las actitudes y el desempeño. Asimismo, los niños españoles presentaron actitudes más positivas y un mayor autoconcepto frente a esta actividad que los finlandeses.

Por último, en el caso del alumnado chileno, según un reporte PISA (OEDC, 2011), si bien sus niveles de motivación por la lectura son cercanos al promedio de la OCDE, existe una disminución de la lectura como pasatiempo entre los años 2000 y 2009 y del tiempo que se invierte en ella. De hecho, un $37 \%$ señala no leer nunca por placer, lo que resulta alarmante si se considera que el disfrute de esta práctica se asocia al mayor desempeño lector y a un alza en los aprendizajes en general (Cunningham \& Stanovich, 1998), por lo que se ahonda la brecha entre lectores y no lectores. Sin embargo, se redujo la cifra de estudiantes que lee solo por obligación y los sujetos leen más ficción. En cuanto a las niñas chilenas, también presentan una mayor motivación por la lectura. Por otra parte, de acuerdo con Valenzuela, Vera y Sotomayor (2015), el compromiso lector de los estudiantes chilenos en PISA y, especialmente, sus actitudes hacia la lectura son determinantes en sus resultados de comprensión lectora, incluso aún más que las estrategias de lectura.

\section{Hipótesis}

Para la validación del instrumento, se espera identificar la estructura descrita para este, es decir, un modelo de dos factores: Gusto por leer y Facilidad de la lectura. Asimismo, también se proyecta que cada uno de los ítems declarados por la encuesta estén vinculados al factor que 
le corresponde. Respecto de la confiabilidad y consistencia interna, se espera que los instrumentos presenten un alfa de Cronbach cercano o superior a 0,7 .

\section{Objetivo}

El objetivo general de este estudio fue validar y adaptar el instrumento sobre actitudes hacia la lectura en niños de $4^{\circ}, 5^{\circ}$ y $6^{\circ}$ Básico del contexto urbano de la Región de la Araucanía, Chile.

\section{Método}

Como la investigación buscó validar y pilotear el instrumento Encuesta de Actitudes hacia la Lectura (Merisuo-Storm \& Soininen, 2012; GarcíaRodicio et al., 2015), corresponde a un diseño descriptivo y evaluativo de tipo cuantitativo.

\subsection{Muestra}

La muestra de validación estuvo confomada por 1.190 estudiantes (610 de género masculino y 580 de género femenino) de $4^{\circ}, 5^{\circ}$ y $6^{\circ}$ Básico de zonas urbanas de la región de la Araucanía. Nos centramos en esta región debido a que esta zona tiene los mayores índices de pobreza del país, una importante presencia de comunidades mapuches (Chile, Ministerio de Desarrollo Social, 2014) y bajos resultados en mediciones de la calidad de la educación, por lo que es imperativo su desarrollo y poder contar con instrumentos validados en esa realidad.

Dentro del total de 100 escuelas básicas municipales urbanas de la Región de la Araucanía, se consideró el universo total de los 36 establecimientos de este tipo (Ministerio de Educación, 2015 y 2016) que existen en las comunas más pobladas (más de 36.000 habitantes) de esta región al año 2015, estas son, en orden decreciente: Temuco (330.324 habitantes), Villarrica (62.820 habitantes), Angol (51.282 habitantes) y Pucón (36.803 habitantes) (Instituto Nacional de Estadísticas, 2013). En efecto, la población de estos territorios abarca el 56\% del total de habitantes de la Araucanía, por lo que es significativa y representativa de la región. Ciertamente, se seleccionaron estas zonas porque interesa indagar sobre los resultados de estudiantes de realidades urbanas; sin 
embargo, como esta región también tiene una importante presencia de ruralidad (Ministerio de Educación, 2015 y 2016), se desea aislar esta variable y enfocarse en los contextos urbanos de mayor población. Además, para contar con una muestra de validación más robusta era conveniente centrarse en establecimienos urbanos, pues tienen una mayor cantidad de estudiantes, sobre todo considerando que en esta región los cursos, en general, son menos numerosos.

Con respecto a la muestra, fue representativa de las comunas seleccionadas y quedó conformada por el 50\% de este universo, es decir, alrededor de 18 escuelas básicas municipales urbanas de estos territorios. El criterio de selección fue aleatorio y la muestra de tipo probabilístico, por lo que el margen de error según el tamaño de la muestra con respecto a su universo es de $5 \%$ y su nivel de confianza de $95 \%$.

\subsection{Instrumento}

La Encuesta de Actitudes hacia la Lectura es un instrumento que se puede aplicar a niños y niñas de $2^{\circ}$ a $6^{\circ}$ grado y fue creada por Merisuo-Storm y Soininen (2012) (Merisuo-Storm \& Aerila, 2018), basándose en otros instrumentos pioneros en su momento como el Elementary Reading Attitude Survey (ERAS) de McKenna y Kear (1990) y The Reading Activity Inventory (RAI) de Guthrie, McGough y Wigfield (1994). Presenta 28 preguntas acerca de las prácticas de lectura y una escala de tipo Likert pictórica de 4 puntos, cuyos niveles muestran imágenes de osos con los siguientes estados de ánimo: muy contento, contento, aburrido y muy aburrido. Con respecto a sus dimensiones, ostenta dos: la primera, es el Gusto por leer y alude a la motivación por esta tarea y, la segunda, es la Facilidad de lectura y se refiere al autoconcepto y autoeficacia que se tiene como lector. Su tabla de especificaciones es la siguiente:

Tabla 1. Dimensiones y puntajes encuesta de actitudes hacia la lectura.

\begin{tabular}{|l|c|c|c|}
\hline \multirow{2}{*}{\multicolumn{1}{|c|}{ Dimensiones }} & Ítems & \multicolumn{2}{c|}{ Escala } \\
\cline { 2 - 4 } & & Alto & Bajo \\
\hline Gusto por leer & $1-21$ & \multirow{2}{*}{$3-4$ puntos } & 1-2 puntos \\
\hline Facilidad de la lectura & $22-28$ & & \\
\hline
\end{tabular}

A continuación se presenta un fragmento del instrumento: 
Figura 1. Fragmento del instrumento Encuesta de actitudes hacia la lectura.

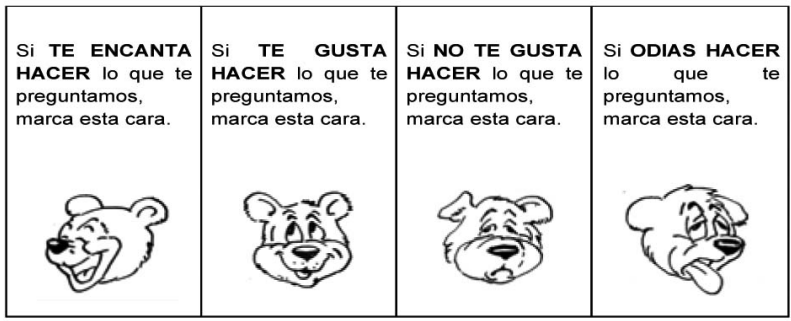

¿Te gusta leer cómics y/o historietas?

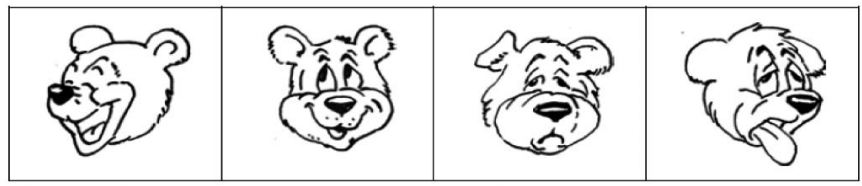

Este instrumento fue aplicado en Finlandia y España con buenos resutados de validación (Villalón et al., 2016; García-Rodicio et al., 2015).

\subsection{Procedimientos}

En primer lugar, la etapa inicial de validación de la encuesta se realizó mediante su adaptación lingüística y cultural al contexto chileno desde la versión traducida al español y aplicada en España (Villalón et al., 2016; García-Rodicio et al., 2015), considerando las expresiones, el léxico y los usos lingüísticos más frecuentes, claros y compresibles en Chile.

En segundo lugar, la próxima fase de la validación correspondió a la revisión del instrumento por parte de tres jueces expertos, quienes enviaron sus evaluaciones, comentarios y sugerencias para su posterior ajuste.

En tercer lugar, se piloteó el instrumento con un grupo de 30 estudiantes de $4^{\circ}, 5^{\circ}$ y $6^{\circ}$ Básico, para observar cómo funcionaba su aplicación y si era necesario realizar ajustes.

Por último, una vez que se modificó el instrumento a partir del dictamen de los jueces y el pilotaje, fue aplicado en formato de lápiz y papel por miembros del equipo de investigación a la muestra total. Cabe señalar que los directores de las instituciones firmaron voluntariamente una carta de autorización, los estudiantes, un asentimiento informado y sus apoderados, una autorización, documentos aprobados por el Comité de Ética UC. Finalmente, se efectuó una validación de contenido, 
constructo y confiabilidad, mediante análisis factorial y del alfa de Cronbach, procedimientos que se describen a continuación.

\section{Resultados}

\subsection{Validación del instrumento}

Se analizó la fiabilidad y validez de constructo del instrumento, en este sentido, los resultados indican que en materia de confiabilidad y validez de constructo la encuesta resulta adecuada para los fines del estudio y se observan las dimensiones declaradas por Merisuo-Storm y Soininen (2012).

\subsubsection{Análisis de fiabilidad de las escalas}

Tabla 2. Fiabilidad escalas Gusto por Leer y Facilidad de Lectura.

\begin{tabular}{|l|c|c|c|c|c|}
\hline Escala & No de sujetos & No de ítems $^{\text {Media }}$ & Desv. Típica & Fiabilidad \\
\hline Gusto por leer & 1148 & 21 & 3,09 & 0,51 & .881 \\
\hline $\begin{array}{l}\text { Facilidad de } \\
\text { lectura }\end{array}$ & 1181 & 7 & 3,24 & 0,49 & .734 \\
\hline
\end{tabular}

La tabla 2 presenta los resultados del análisis de consistencia interna basados en el coeficiente alfa de Cronbach para las escalas Gusto por Leer (21 ítems) y Facilidad de Lectura ( 7 ítems) presentes en la Encuesta de Actitudes hacia la Lectura y la Escritura en niños. Siguiendo la clasificación de George y Mallery (2003) este coeficiente resulta bueno para la escala Gusto por Leer $(\alpha=0,881)$ y aceptable para la escala Facilidad de Lectura $(\alpha=0,734)$.

Tabla 3. Fiabilidad escalas Gusto por Leer y Facilidad de Lectura según nivel educativo.

\begin{tabular}{|c|c|c|c|c|c|c|}
\hline Escala & $\begin{array}{l}\text { Nivel } \\
\text { Educativo }\end{array}$ & $\begin{array}{l}\text { No de } \\
\text { sujetos }\end{array}$ & $\begin{array}{l}\text { No de } \\
\text { ítems }\end{array}$ & Media & $\begin{array}{l}\text { Desv. } \\
\text { Típica }\end{array}$ & Fiabilidad \\
\hline \multirow[t]{3}{*}{ Gusto por leer } & $4^{\circ}$ básico & 399 & 21 & 3,23 & 0,66 & .887 \\
\hline & $5^{\circ}$ básico & 381 & 21 & 3,03 & 0,73 & .864 \\
\hline & $6^{\circ}$ básico & 368 & 21 & 3,01 & 0,88 & .879 \\
\hline \multirow{3}{*}{$\begin{array}{l}\text { Facilidad de } \\
\text { lectura }\end{array}$} & $4^{\circ}$ básico & 407 & 7 & 3,54 & 0,74 & .741 \\
\hline & $5^{\circ}$ básico & 402 & 7 & 3,52 & 0,77 & .697 \\
\hline & $6^{\circ}$ básico & 372 & 7 & 3,56 & 0,83 & .762 \\
\hline
\end{tabular}


Por su parte, la tabla 3 presenta los resultados del análisis de consistencia interna basados en el coeficiente alfa de Cronbach para las escalas Gusto por Leer (21 ítems) y Facilidad de Lectura ( 7 ítems) aplicadas a cada uno de los diferentes niveles educativos. Se destaca en este aspecto la baja magnitud del coeficiente alfa de Cronbach para la escala Facilidad de lectura administrada al nivel 6º́sico $(\alpha=, 697)$, siendo un coeficiente cuestionable según la clasificación de George y Mallery (2003). En relación con esto último y para escalas con menos de 10 ítems, Loewenthal (1996) sugiere que un valor de fiabilidad superior a 6 es considerado aceptable.

\subsubsection{Validez de constructo mediante análisis factorial exploratorio}

En primer lugar, previo a la realización del análisis factorial, se analizó la medida de adecuación muestral $\mathrm{KMO}=0,911$, que supera el límite de aceptabilidad de 0,5. El test de esfericidad de Bartlett presentó una magnitud $\chi^{2}=8670,702 \mathrm{p}=0,000$. De este modo, tanto por tamaño muestral y correlación entre variables procedió la realización del análisis factorial. A través del método de Análisis de Componentes Principales se extrajeron 7 factores con autovalores $\geq 1$, explicándose el 54,45\% de la varianza acumulada. 
Tabla 4. Matriz de componentes rotados.

\begin{tabular}{|c|c|c|c|c|c|c|c|}
\hline & \multicolumn{7}{|c|}{ Factor } \\
\hline ITEM & 1 & 2 & 3 & 4 & 5 & 6 & 7 \\
\hline p1_AALL & ,697 & ,252 & , 089 & 148 & 165 &,- 054 &,- 024 \\
\hline p2_AALL & 240 & ,341 & ,080 & 161 &,- 034 & ,625 &,- 092 \\
\hline p3_AALL & 274 & ,255 & ,033 &,- 054 & ,277 &,- 203 & , 476 \\
\hline p4_AALL & ,664 & , 072 & ,093 & , 067 & , 047 & 116 & 160 \\
\hline p5_AALL &,- 051 & 174 & ,541 &,- 051 & , 054 & -,002 & 108 \\
\hline p6_AALL & ,660 & ,332 & , 075 & ,012 & 133 &,- 001 & ,036 \\
\hline p7_AALL & 163 & ,460 & 203 & 143 &,- 033 &,- 098 & 299 \\
\hline p8_AALL & ,716 & ,021 & , 072 & ,035 & , 078 & ,093 & 151 \\
\hline p9_AALL & ,166 & ,706 & ,164 & , 067 & 147 & ,058 & ,010 \\
\hline p10_AALL & ,045 & ,676 & ,188 & ,146 & , 054 & ,326 &,- 006 \\
\hline p11_AALL & ,405 & ,496 & 150 &,- 010 &,- 008 & ,085 & , 097 \\
\hline p12_AALL & ,306 & ,578 & 189 &,- 003 & 203 & 182 & ,014 \\
\hline p13_AALL & ,473 & 103 & ,169 & ,016 & ,215 & ,489 & 113 \\
\hline p14_AALL & ,310 & ,227 & 299 &,- 092 & 288 & ,349 & , 081 \\
\hline p15_AALL &, 514 & ,298 & , 078 & ,051 & , 075 & ,270 &,- 080 \\
\hline p16_AALL & ,457 & , 068 & , 410 &,- 010 & 239 & 197 & ,246 \\
\hline p17_AALL & 119 & 177 & ,722 & ,081 & ,035 & 161 &,- 026 \\
\hline p18_AALL & ,401 & ,071 & ,472 & ,231 & 112 &,- 094 &,- 253 \\
\hline p19_AALL & ,400 & 104 & ,412 &,- 011 & ,227 & ,346 & 170 \\
\hline p20_AALL & ,196 & 139 & ,693 & ,102 &,- 006 & ,012 & ,085 \\
\hline p21_AALL & 128 & , 008 & 115 & 121 &,- 046 & 101 &, 769 \\
\hline p22_AALL & ,192 & ,010 & 110 &, 705 & ,163 &,- 285 &,- 092 \\
\hline p23_AALL &,- 011 & 139 & , 061 & ,704 & ,030 & ,289 & ,012 \\
\hline p24_AALL & , 077 & ,022 & ,036 & ,660 & ,250 &,- 043 & ,107 \\
\hline p25_AALL &,- 034 & 110 &,- 045 & ,606 & 185 &, 417 & ,142 \\
\hline p26_AALL & ,175 & ,197 & ,031 & ,217 & ,692 &,- 044 &,- 029 \\
\hline p27_AALL & ,026 & , 078 & , 080 & ,258 & ,693 & ,043 & 111 \\
\hline p28_AALL & ,196 &,- 010 & ,061 & ,084 & ,679 & ,134 &,- 043 \\
\hline
\end{tabular}

La Tabla 4 presenta la matriz de componentes rotados mediante rotación Varimax con normalización Kaiser, mostrando en el texto destacado las cargas factoriales con magnitud $\geq 0,3$ para cada uno de los ítems que componen la Encuesta de Actitudes Hacia la Lectura y Escritura. De este modo, es posible señalar que 12 ítems presentan 
cargas de magnitud asociadas al factor 1 , siete ítems presentan cargas de magnitud asociadas al factor 2 , seis ítems presentan cargas de magnitud asociadas al factor 3 , cuatro ítems presentan cargas de magnitud asociadas al factor 4, tres ítems presentan cargas de magnitud asociadas al factor 5, seis ítems presentan cargas de magnitud asociadas al factor $6 \mathrm{y}$ dos ítems presentan cargas de magnitud asociadas al factor 7 . Además, se destaca en el análisis que en los factores 1, 2, 3 y 7 solamente se observan cargas de magnitud asociadas a los ítems comprendidos entre el ítem 1 (p1_AALL) y el ítem 21 (p21_AALL), que en los factores 4 y 5 solo cargan de forma considerable los ítems comprendidos entre el ítem 22 (p22_AALL) y el ítem 28 (p28_AALL). Así, solamente en el factor 6 se encuentran ítems que presentan cargas superiores a 0,3 antes o después del ítem 21.

Sin embargo, al analizar el gráfico de sedimentación, se puede identificar ya un punto de inflexión a partir de los factores 2 o 3.

Gráfico 1. Gráfico de sedimentación.

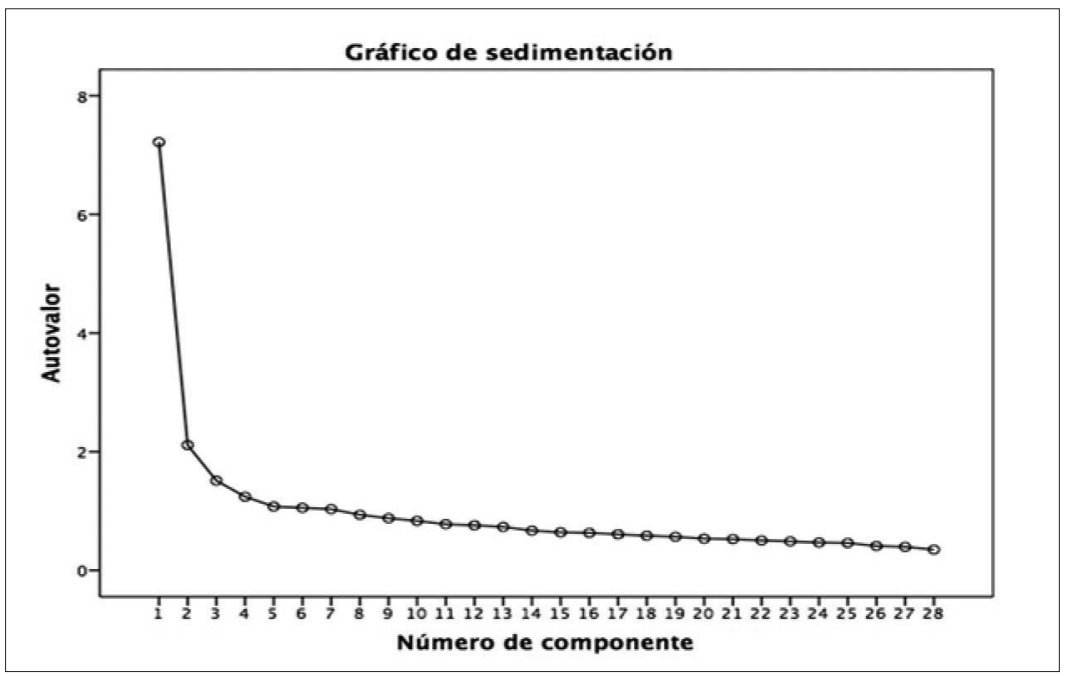

En consecuencia, la realización del Análisis Factorial Exploratorio indica que a pesar de existir 7 factores con autovalores $\geq 1$, el gráfico de sedimentación permite aventurar, en función de los criterios de análisis, la existencia de menos factores, 2 o 3 presumiblemente. 
6.1.3. Validez de constructo mediante análisis factorial confirmatorio

En primer lugar, antes de la realización del análisis factorial, se analizó la medida de adecuación muestral KMO = 0,911 que supera el límite de aceptabilidad de 0.5. Asimismo, el test de esfericidad de Bartlett presentó una magnitud $\chi^{2}=8670,702 \mathrm{p}=0,000$. De esta manera, tanto por tamaño muestral y correlación entre variables procedió la realización del análisis factorial.

De acuerdo con la estructura teórica del instrumento, este se encuentra conformado por dos escalas o dimensiones: "Gusto por leer" (ítem 1 al ítem 21) y "Facilidad de Lectura” (ítem 22 al ítem 28), por tanto, se efectuó Análisis Factorial Confirmatorio, considerando la extracción de dos factores. Lo anterior se realizó a través del método de Análisis de Componentes Principales y rotación Varimax.

A partir del análisis, los factores extraídos (2) dan cuenta del 33,32\% de la varianza, y la carga factorial de cada uno de los ítems de la encuesta se presentan en la siguiente tabla, destacándose las cargas con magnitud $\geq 0,3$. 
Tabla 5. Matriz de componentes rotados.

\begin{tabular}{|c|c|c|}
\hline & \multicolumn{2}{|c|}{ Factor } \\
\hline ITEM & 1 & 2 \\
\hline p1_AALL & ,615 & ,215 \\
\hline p2_AALL & ,485 & ,177 \\
\hline p3_AALL & ,386 & 100 \\
\hline p4_AALL &, 581 & ,086 \\
\hline p5_AALL &, 313 &,- 009 \\
\hline p6_AALL & ,658 & ,094 \\
\hline p7_AALL &, 417 & 120 \\
\hline p8_AALL & ,583 & ,071 \\
\hline p9_AALL &, 543 & ,178 \\
\hline p10_AALL & ,504 & ,212 \\
\hline p11_AALL & ,612 & ,017 \\
\hline p12_AALL & ,638 & 147 \\
\hline p13_AALL & ,616 & 161 \\
\hline p14_AALL & ,600 & ,107 \\
\hline p15_AALL &, 585 & 113 \\
\hline p16_AALL & ,645 & 128 \\
\hline p17_AALL &, 513 & 100 \\
\hline p18_AALL & ,433 & ,243 \\
\hline p19_AALL & ,650 & 134 \\
\hline p20_AALL & ,506 & ,083 \\
\hline p21_AALL & ,275 & ,082 \\
\hline p22_AALL & , 028 & ,658 \\
\hline p23_AALL & ,083 &, 636 \\
\hline p24_AALL & ,037 & ,686 \\
\hline p25_AALL & ,093 & ,644 \\
\hline p26_AALL & ,268 &, 558 \\
\hline p27_AALL & ,173 &, 593 \\
\hline p28_AALL & 257 & ,439 \\
\hline
\end{tabular}

Según la Tabla 5, es posible apreciar que la totalidad de los ítems comprendidos entre el ítem 1 y el ítem 20 presentan cargas factoriales de magnitud $\geq 0,3$, situación que se relaciona de muy buena manera con la descripción del instrumento, pues los primeros 21 ítems se vinculan con el gusto que genera en el estudiante llevar a cabo algunas actividades 
asociadas a la lectura y la escritura. De forma análoga, los ítems comprendidos entre el ítem 22 e ítem 28 presentan cargas factoriales de magnitud superior a 0,3 solamente en su relación con el factor 2 , vale decir, ítems que consultan acerca de la facilidad que percibe el estudiante en su relación con las tareas de lectura. Asimismo, es necesario destacar que el ítem 21 ( “ $i$ Te gusta leer textos en Internet o en el computador o el tablet?") no se manifiesta con fuerza en ninguno de estos dos factores principales.

El siguiente gráfico refleja la posición de cada ítem en el espacio rotado.

Gráfico 2. Cargas factoriales de los ítems.

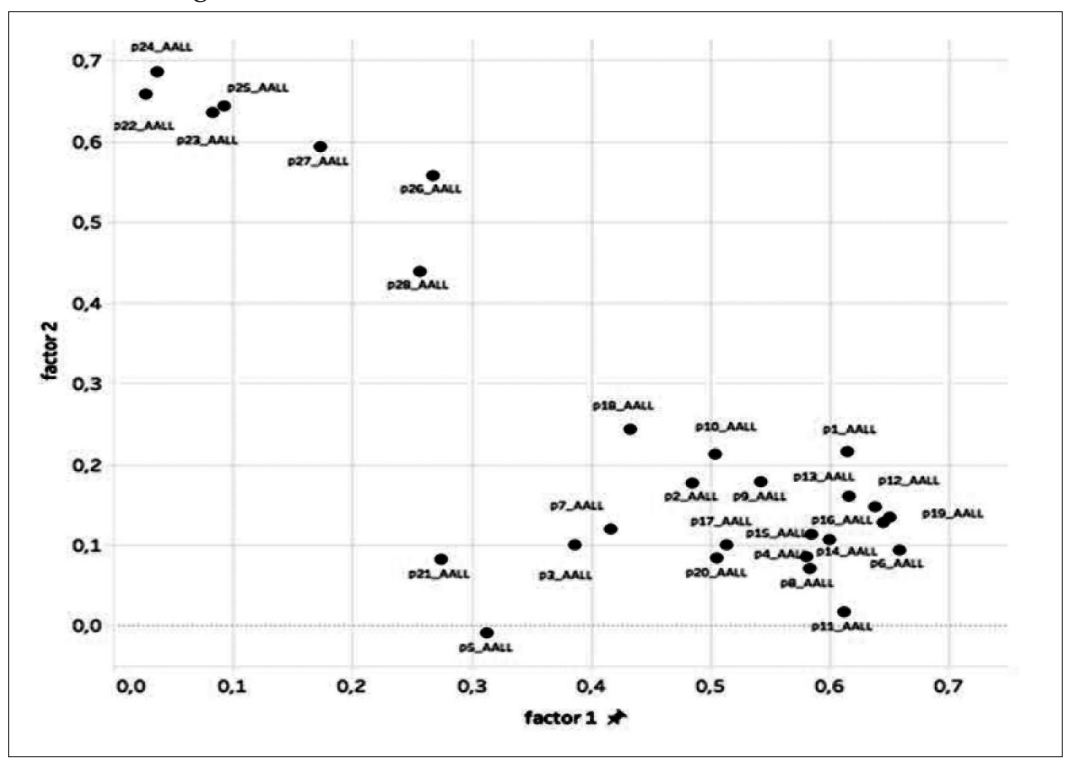

De acuerdo con el gráfico 2, se reafirma el análisis presente en la tabla 5, es decir, al considerar la existencia de dos dimensiones el Análisis Factorial Comprobatorio es capaz de identificar las dos dimensiones teóricas subyacentes en la encuesta: "Gusto por leer", asociada al factor 1 y "Facilidad de Lectura" asociada al factor 2. Por otro lado, y en relación al ítem 21, si bien su carga factorial es baja en relación a cada uno de los factores, se encuentra más próximo a la agrupación de ítems correspondientes al factor 1 que a los ítems correspondientes al factor 2. Un último elemento a destacar es que los ítems no poseen cargas factoriales de magnitud similar para cada uno 
de los dos factores simultáneamente. En efecto, en términos generales, a medida que un ítem se acerca más a un factor deja de pertenecer gradualmente a otro.

En consecuencia, para la aplicación futura del instrumento y a partir de los análisis y la validación llevada a cabo, se considerará un modelo con todos los ítems a excepción del 21, cuyo enunciado explora el nivel de gusto por la lectura de textos en Internet, en el computador o en otros dispositivos tecnológicos como celulares o tabletas. Si bien, en Chile, el uso de aparatos tecnológicos se ha masificado, probablemente esta muestra de estudiantes de educación básica aún no asocia la lectura al uso de la tecnología, pues quizás la han utilizado solo con fines de entretención audiovisual, como usuarios de videojuegos o de redes sociales, pese a que en estas últimas también leen y escriben, por lo que seguramente existe un divorcio entre estas actividades de lectura y escritura y aquellas que realizan en la escuela. Además, es necesario recordar que los sujetos de la muestra corresponden a estudiantes de escuelas públicas de la Región de la Araucanía, zona que -como ya se ha mencionado- presenta los mayores índices de pobreza del país (Chile. Ministerio de Desarrollo Social, 2014), por lo que posiblemente no tengan tanto acceso a dispositivos tecnológicos y a Internet como en otras regiones u otro tipo de establecimientos educativos. No obstante, será necesario continuar indagando en las razones que expliquen el comportamiento de este ítem, especialmente porque, según Agencia de la Calidad de la Educación (2016), existe algún grado de vínculo entre la motivación lectora y la frecuencia de lectura de textos digitales.

\section{Conclusiones}

A partir de los resultados de validación de la Encuesta de Actitudes hacia la Lectura y Escritura, fue posible confirmar satisfactoriamente las dimensiones del instrumento y de los ítems asociados a cada una de ellas, por tanto, se cumplió el objetivo del estudio y se constataron sus hipótesis, si bien será necesario revisar y analizar con profundidad la situación del ítem 21 y eliminarlo. Esto permitiría, a nuestro juicio, establecer que es un instrumento confiable para evaluar las actitudes hacia la lectura en estudiantes de $4^{\circ}, 5^{\circ}$ y $6^{\circ}$ año básico para sectores urbanos de la región de La Araucanía, Chile. De esta forma, se podrá contar con un instrumento que entregue información fiable y que pueda contribuir en alguna me- 
dida a entregar más información acerca de variables que pueden influir en los resultados de lectura de los estudiantes.

Como se ha señalado, hay abundante literatura sobre la influencia de las actitudes de lectura en el desempeño lector, no obstante, es justamente este factor el que hasta ahora ha sido desatendido en la educación chilena. Por lo tanto, las futuras políticas públicas y las medidas e intervenciones educativas deberían también abordar este factor de modo central, pues posiblemente sea la clave para desarrollar la comprensión lectora, el rendimiento general y los aprendizajes de los estudiantes del sistema escolar. Especialmente si consideramos que las actitudes de lectura han demostrado ser más relevantes en el desempeño que las estrategias de lectura (Valenzuela et al., 2015).

Con respecto a las limitaciones de este estudio, en primer lugar, si bien la muestra es representativa de la Región de la Araucanía, solo se centró en contextos urbanos y las comunas más pobladas de la región por contar con un mayor volumen de población, por ende, los resultados no son extendibles a la realidad de los establecimientos escolares rurales, los cuales son numerosos en esta región (Ministerio de Educación, 2015 y 2016). En segundo lugar, la investigación solo se enmarcó en la Región de la Araucanía, por lo que se describió la realidad de los alumnos de este territorio, que por sus particularidades puede ser distinta del resto del país. En tercer lugar, el alumnado que se consideró en el estudio es de $4^{\circ}$, $5^{\circ}$ y $6^{\circ}$ Básico, para así disponer de un instrumento que se pueda utilizar para comparar sus resultados con aquellos arrojados por la evaluación Simce de Lectura y Escritura, de este modo, no se contemplaron cursos inferiores ni superiores.

Finalmente, respecto de las proyecciones de este estudio, los resultados de la aplicación de este instrumento permitirán diseñar acciones, intervenciones, recomendaciones y herramientas didácticas a nivel de los establecimientos educativos como de las políticas públicas y de los programas de formación docente, con el fin de producir el cambio conceptual necesario en la enseñanza para elevar las actitudes y el placer por la lectura del estudiantado y, por ende, la calidad de sus aprendizajes. En este sentido, consideramos que la implementación de planes de lectura y escritura a traves del currículum y de las disciplinas en los contextos escolares y de formación inicial docente, puede ser una alternativa efectiva para generar mayor motivación por las prácticas de lectura de diversos géneros discursivos (Bazerman, 
Bethel, Chavkin, Fouquette \& Garufis, 2005; Carlino, 2005; Street \& Street, 1995; Tolchinsky \& Simó, 2001). Asimismo, el abordaje de las actitudes de lectura de los docentes y su rol como mediadores de lectura, de acuerdo con la Política Nacional de la Lectura y el Libro (Consejo Nacional de la Cultura y las Artes, 2015), parece ser un factor clave en el desarrollo de las prácticas lectoras del alumnado y debe ser considerado por el currículum escolar (Ministerio de Educación, 2012) y los establecimientos educativos.

\section{Referencias bibliográficas}

Agencia de la Calidad de la Educación. (2016). Estudio: factores que influyen en la motivación por la lectura y su relación con logros de aprendizajes e indicadores de desarrollo personal y social. Santiago: ACE.

Alexander J. E. \& Filler R. C. (1976). Attitudes and reading. Newark, DE: International Reading Association.

Azjen, I. \& Fishbein, M. (1977). Attitude-behavior relations: A theoretical analysis and review of empirical research. Psychological Bulletin, 84, 888-918.

Bazerman, C., Bethel, L., Chavkin, T., Fouquette, D. \& Garufis, J. (2005). Reference guide to writing across the curriculum. West Lafayette: Parlor Press and The WAC Clearinghouse.

Becerra-Lubies, R. \& Mayo González, J. S. (2015). Percepciones acerca del rol de las comunidades mapuche en un jardín intercultural bilingüe. Psicoperspectivas, 14(3), 56-67. doi:10.5027/ PSICOPERSPECTIVAS-VOL14-IS- SUE3-FULLTEXT-606.

Calderón López, M. (2015). Producción escrita y literacidad familiar. Literatura y lingüistica, 32, 259-282. Recuperado de http:// www.scielo.cl/pdf/lyl/n32/art14.pdf.

Carlino, P. (2005). Escribir, leer y aprender en la universidad. Una introducción a la alfabetización académica. México: Fondo de Cultura Económica.

Carpi, A. \& Breva, A. (2001). La predicción de la conducta a través de los constructos que integran la teoría de acción planeada. Revista electrónica de motivación y emoción, 4(7), 1-17. 
Cervini, R. (2002). Desigualdades en el logro académico y reproducción cultural en Argentina. Revista Mexicana de Investigación Educativa, 7(16), 445-500.

Chambers, A. (2007). El ambiente de la lectura. México: Fondo de Cultura Económica.

Chile. Agencia de Calidad de la Educación. (2017a). Estudio Internacional de Lectura PIRLS 2016: Presentación Nacional de Resultados. Recuperado de http://www.agenciaeducacion.cl/noticias/14-estudiantes-chile-logran-los-mejores-resultados-estudiointernacional-lectura/.

Chile. Agencia de Calidad de la Educación. (2017b). Resultados Educativos 2016. IX Región de la Araucanía. Recuperado de http://archivos.agenciaeducacion.cl/PDFConferencia_ ARAUCANIA_2016.pdf.

Chile. Ministerio de Desarrollo Social. (2014). Encuesta de Caracterización Socioeconómica Nacional Casen 2013. Recuperado de http://www.ministeriodesarrollosocial.gob. cl/resultados-encuesta-casen-2013/.

Consejo Nacional de la Cultura y las Artes. (2015). Política Nacional de la Lectura y el Libro 2015-2020. Santiago: CNCA.

Consejo Nacional de la Cultura y las Artes. (2014). Encuesta de comportamiento lector. Santiago: CNCA.

Coles, M. \& Hall, C. (2002). Gendered readings: Learning from children's reading choices. Journal of Research in Reading, 25, 96-108. doi: http://dx.doi.org/10.1111/1467-9817.00161.

Cunningham, A. E. \& Stanovich, K. E. (1998). What reading does for the mind. American educator, 22, 8-17.

Daisey, P. (2009). The reading experiences and beliefs of secondary preservice teachers. Reading Horizons, 49(2), 167-190.

Eccles, J. S. \& Wigfield, A. (2002). Motivational beliefs, values and goals. Annual Review of Psychology, 53, 109-132. Doi: https://doi. org/10.1146/annurev.psych.53.100901.135153.

Errázuriz, M. C., Becerra, R., Cocio, A., Davison, O. \& Fuentes, L. (2018). Concepciones sobre lectura de profesores en ejercicio: Perfiles lectores de docentes y su relación con el desempeño de sus estudiantes. Informe final FONIDE $N^{\circ}$ FX11619. Santiago: Ministerio de Educación. 
Fuentes, L. (2009). Diagnóstico de comprensión lectora en educación básica en Villarrica y Loncoche, Chile. Perfiles educativos, 31(125), 23-37.

Fundación La Fuente. (2016). No es un cuento: lo que los apoderados y profesores piensan sobre la lectura infantil (vol.2). Santiago: Fundación La Fuente.

García-Rodicio, H., Izquierdo-Magaldi, B., Fernández-Gutiérrez, A. \& Villalón, R. (2015), ¿Importa la actitud hacia la lectura a la hora de comprender un texto?. Ponencia presentada en 3rd International Congress of Educational Sciences and Development, San Sebastián, España.

George, F. \& Mallery, P. (2003). SPSS for Windows step by step: A Simple Guide and Reference. 11.0 Update (4.a ed.). Boston: Allyn \& Bacon.

Gambrell, L. B., Palmer, B. M., Codling, R. M. \& Mazzoni, S. A. (1996). Assessing motivation to read. The Reading Teacher, 49, 518-533. Doi: https://doi.org/10.1598/RT.49.7.2.

Granado, C. (2014). Teachers as readers: a study of the reading habits of future teachers. Culture and Education, 26(1), 44-70. Doi: 10.1080/11356405.2014.908666.

Guthrie, J. T., Hoa, A. L. W., Wigfield, A., Tonks, S. M., Humenick, N. M. \& Littles, E. (2007). Reading motivation and reading comprehension growth in the later elementary years. Contemporary Educational Psychology, 32(3), 282-313.

Guthrie. J., McGough, K. \& Wigfield, A. (1994). Measuring reading activity: An Inventory (Instructional Resource No 4). Athens, GA: Universities of Georgia and Maryland, National Reading Research Center.

Instituto Nacional de Estadísticas, Dirección Regional de La Araucanía. (2013). Compendio estadístico regional la Araucanía Informe Anual 2013. Santiago: INE.

Jiménez, J. E., Rodríguez, C., Suárez, N., O’Shanahan, I., Villadiego, Y., Uribe, C., Villalobos, J. A. \& Rodas, P. (2015). Teachers' implicit theories of learning to read: A cross-cultural study in Ibero-American countries. Reading and Writing, 28(9), 1355-1379. 
Latorre, M. (2014). Estudio Mis lecturas diarias y valoracion de la lectura $5^{\circ}$ a $8^{\circ}$ Básico. Santiago: Ministerio de Educación.

Lockwood, M. (2011). Promover el placer de leer en la educación primaria. Madrid: Morata.

Loewenthal, K. M. (1996). An introduction to psychological tests and scales. London: UCL Press Limited.

Logan, S. \& Johnston, R. S. (2010). Investigating gender differences in reading. Educational Review, 62, 175-187. doi: http://dx.doi. org/10.1080/o0131911003637006.

McKenna, M. C., Kear, D. J. \& Ellsworth, R. A. (1995). Children's attitudes toward reading: A national survey. Reading Research Quarterly, 30(4), 934-956.

McKenna, M. C. \& Kear, D.J. (1990). Measuring attitude toward read: A new tool for teachers. The Reading Teacher, 43, 626-639.

Martínez, R. S., Aricak, O. T. \& Jewell, J. (2008). Influence of reading attitude on reading achievement: A test of the temporal-interaction model. Psychology in the Schools, 45(10), 1010-1023.

Mathewson, G. C. (1994). Model of attitude influence upon reading and learning to read. En H. Singer \& R. B. Ruddell (Eds.), Theoretical models and processes of reading (4th ed., pp. 841-859). Newark, DE: International Reading Association.

Merisuo-Storm, T. \& Aerila, J. (2018). Boys' and Girls' Reading Skills and Attitudes During the First Six School Years. En P. Orellana \& P. Baldwin (Eds.), Reading Achievement and Motivation in Boys and Girls. Field Studies and Methodological Approaches (pp. 157 -181). Cham: Springer.

Merisuo-Storm, T. \& Soininen, M. (2012). Young boys' opinions about reading, literacy lessons and their reading competence. En ICERI2012 Proceedings (pp. 4109-4118). Madrid, España: IATED Publications.

Ministerio de Educación. (2016). Estadisticas de la Educación 2015. Santiago: Mineduc.

Ministerio de Educación. (2015). Esquema de registro bases Sistema Nacional de Evaluación del Desempeño (SNED) 2014-2015. Santiago: Mineduc. 
Morrison, T. G., Jacobs, J. \& Swinyard, W. R. (1999). Do teachers who read personally use recommended practices in their classroom? Reading Research and Instruction, 38, 81-100.

Mujica, A., Guido, P. \& Mercado, J.S. (2011). Actitudes y conducta lectora: una aplicación de la teoría de la conducta planeada en estudiantes de nivel medio superior. Liberabit, 17(2),171-178.

Mullis, I. V. S., Martin, M. O., Kennedy, A. M. \& Foy, P. (2007). PIRLS 2006 international report: IEA's progress in international reading literacy study in primary schools in 40 countries. Chestnut Hill, MA: Boston College.

Muñoz, C., Ferreira, S., Sánchez, P., Santander, S., Pérez, M. \& Valenzuela, J. (2012). Características psicométricas de una escala para caracterizar la motivación por la lectura académica. Revista Electrónica de Investigación Educativa, 14(2), 118-132. Recuperado de http://www.scielo.org.mx/pdf/redie/v14n2/ v14n2a9.pdf

Navarro, M., Orellana, P. \& Baldwin, P. (2018). Validación de la Escala de Motivación Lectora en Estudiantes Chilenos de Enseñanza Básica. Psykhe, 27(1), 1-17.

OECD. (2014). Resultados PISA 2012 en foco: Lo que el alumnado saben a los 15 años de edad y lo que pueden hacer con lo que saben. Recuperado de https://www.oecd.org/pisa/keyfindings/ PISA2012 Overview ESP-FINAL.pdf

OECD (Ed.). (2011). PISA: Do students today read for pleasure? PISA in focus, (8), 1-4.

Orellana, P. (2018). To What Extent Is Reading Motivation a Significant Predictor of Reading Achievement when Controlling for Language and Cognitive Ability? A Systematic Review. En P. Orellana \& P. Baldwin (Eds.), Reading Achievement and Motivation in Boys and Girls. Field Studies and Methodological Approaches (pp. 79-96). Cham: Springer.

Petscher, Y. (2010). A meta-analysis of the relationship between student attitudes towards reading and achievement in reading. Journal of Research in Reading, 33, 335-355. doi:10.1111/j.1467-9817.2009.01418.x.

Schaffner, E., Schiefele, U. \& Ulferts, H. (2013). Reading amount as a mediator of the effects of intrinsic and extrinsic reading motivation 
on reading comprehension. Reading Research Quarterly, 48, 369-385. doi: http://dx.doi.org/10.1002/rrq.52

Street, J. C. \& Street, B. V. (1995). The schooling of literacy. En P. Murphy, M. Selinger, J. Bourne \& M. Briggs (Eds.), Subject Learning in the Primary Curriculum: Issues in English, Science and Mathematics (pp. 72-86). London: Routledge.

Stutz, F., Schaffner, E. \& Schiefele, U. (2016). Relations among reading motivation, reading amount, and reading comprehension in the early elementary grades. Learning and Individual Differences, 45, 101-113. Doi: http://dx.doi.org/10.1016/j.lindif.2015.11.022

Tolchinsky, L. \& Simó, R. (2001). Escribir y leer a través del currículum. Barcelona: Horsori.

Valenzuela, J. P., Vera, G. G. \& Sotomayor, C. (2015). The role of reading engagement in improving national achievement: An analysis of Chile's 2000-2009 PISA results. International Journal of Educational Development, 40, 28-39.

Van Schooten, E., De Glopper, K. \& Stoel, R. D. (2004). Development of attitude toward reading adolescent literature and literary reading behavior. Poetics, 32(5), 343-386.

Villalón, R., Merisuo-Storm, T., Izquierdo-Magaldi, B., Melero., A. \& Soininen, M. (2016). Reading attitudes and text comprehension of Finnish and Spanish pupils: a comparative study. Ponencia presentanda en Conference of the EARLI SIG 2 Comprehension of Text and Graphics, Geneva, Switzerland.

Wigfield, A., Guthrie, J. T., Perencevich, K. C., Taboada, A., Klauda, S. L., McRae, A. \& Barbosa, P. (2008). Role of reading engagement in mediating effects of reading comprehension instruction on reading outcomes. Psychology in the Schools, 45(5), 432-445. 


\section{ANEXO}

\section{Ítems de la Encuesta de Actitudes hacia la Lectura y la Escritura}

1. ¿Te gusta leer?

2. ¿Te gusta escribir?

3. ¿Te gusta leer cómics y/o historietas?

4. ¿Te gusta que te regalen libros?

5. ¿Te gusta que te lean en voz alta?

6. ¿Te gusta leer cuentos?

7. ¿Te gusta leer libros o textos que digan cómo son las cosas: animales, deportes, lugares, personas, etc.?

8. ¿Te gusta ir a la biblioteca de tu escuela o de tu comuna?

9. ¿Te gustó lo que tuviste que hacer en la escuela para aprender a leer?

10. ¿Te gustó lo que tuviste que hacer en la escuela para aprender a escribir?

11. ¿Te gustó leer esos libros que usaste para aprender las letras?

12. ¿Te gustó hacer actividades en clase de Lenguaje para aprender a leer y escribir?

13. ¿Te gusta escribir sobre lo que has leído?

14. ¿Te gusta hacer otras actividades sobre lo que has leído?

15. ¿Te gusta hacer las tareas de leer y escribir que tu profesora o profesor te manda para la casa?

16. ¿Te gusta hablar con tus compañeros sobre los libros y textos que estás leyendo?

17. ¿Te gusta hacer las tareas de leer y escribir junto con algún compañero?

18. ¿Te gusta leer en voz alta en clase?

19. ¿Te gusta comentar con otros compañeros un libro o texto que has leído (por ejemplo, si te ha gustado o no y porqué)?

20. ¿Te gusta leer junto con otro compañero?

21. ¿Te gusta leer textos en Internet o en el computador o el tablet?

22. ¿Te resulta fácil leer?

23. ¿Te resulta fácil escribir?

24. ¿Has aprendido fácilmente a leer? 
25. ¿Has aprendido fácilmente a escribir?

26. ¿Es fácil para ti entender lo que lees en la escuela?

27. ¿Es fácil para ti entender las palabras que lees?

28. ¿Te resulta fácil acordarte de lo que lees? 